\title{
Autômatos Celulares e Sistema Multiagente para Simulação da Propagação de Doenças
}

\section{Cellular Automata and Multi-Agent System for Simulation of Disease Spread}

\author{
Rogério Luis Rizzi ${ }^{1,2}$ \\ Claudia Brandelero Rizzi ${ }^{3,4}$ \\ Wesley Luciano Kaizer ${ }^{5,6}$ \\ Pétterson Vinicius Pramiu 7, 8
}

Data de submissão: 16/06/2016, Data de aceite: 22/10/2016

\begin{abstract}
Resumo: Neste trabalho é apresentado um estudo sobre modelos computacionais epidemiológicos enfocando o tipo Suscetível-Infectado-Removido, SIR, e diferentes estratégias de solução à simulação computacional da propagação de doenças transmissíveis. Mostra-se que modelos baseados em autômatos celulares tipo Lattice Gas Cellular Automata, LGCA, têm soluções assemelhadas às obtidas por sistemas multiagentes, diferentemente de autômatos celulares difusivos. Os resultados obtidos da literatura, bem como aqueles decorrentes deste trabalho, apontam que tais metodologias têm potencial à simulação da dinâmica de fenômenos ecológicos, biológicos e físicos que possam assim ser modelados. Indicam, contudo, que resultados mais condizentes com dados reais dependem do desenvolvimento e da parametrização de modelos que adicionem características essenciais ao fenômeno, como a interação entre indivíduos e meio ambiente, e a heterogeneidade do sistema relacional de contatos.
\end{abstract}

Palavras-chave: Autômatos celulares, LGCA, Multiagente, Epidemiologia Computacional

\footnotetext{
${ }^{1}$ Prof. Associado do Centro de Ciências Exatas e Tecnológicas da Universidade Estadual do Oeste do Paraná, Unioeste - CEP:85819-110, Cascavel Paraná, Brasil.

2 EMAIL $\}$

${ }^{3}$ Profa. Associado do Centro de Ciências Exatas e Tecnológicas da Universidade Estadual do Oeste do Paraná, Unioeste - CEP:85819-110, Cascavel Paraná, Brasil.

${ }^{4}$ EMAIL $\}$

${ }^{5}$ Graduando em Ciência da Computação da Universidade Estadual do Oeste do Paraná, Unioeste - Cascavel Paraná, Brasil.

${ }^{6}\{$ EMAIL $\}$

${ }^{7}$ Doutorando do Instituto de Ciências da Computação e Matemática Computacional da Universidade de São Paulo, ICMC/USP - CEP:13566-590, São Carlos, São Paulo, Brasil.

8 ppramiu@gmail. com\}
} 


\begin{abstract}
This paper presents a study of computational epidemiological models, focusing models types Susceptible-Infected-Removed, discussing some strategy of solution for computational simulation of the spread of hypothetical communicable diseases. It is shown that models based on cellular automata type Lattice Gas Cellular Automata has likened the solution obtained by multi-agent system, unlike models in diffusive cellular automata. The results of the literature as well as those resulting from this work, point out that such an approach can simulate the dynamics of a variety of ecological, biological and physical phenomena. But also indicate that more consistent results with actual data may depend the development of models that add other features, such as interaction between individuals and the environment and the heterogeneity of the relational system of contacts.
\end{abstract}

Keywords: Cellular Automata, LGCA, Multi-Agente System, Computational Epidemiology

\title{
1 Introdução
}

As primeiras considerações epidemiológicas remontam ao século XIX e foram conduzidas por John Snow, médico londrino que detectou a fonte de surto epidêmico de cólera, por meio da análise dos ambientes espaciais e sociais dos indivíduos infectados. Atualmente análises preliminares são realizadas para avaliar riscos epidemiológicos. Entidades governamentais ou organizações de saúde têm interesse em quantificar indivíduos potencialmente afetados pela doença, para gestão ou tomadas de decisão em termos de saúde [31].

Modelos computacionais baseados em indivíduos vêm sendo empregados para estudar a propagação e a transmissão de doenças, que são processos centrais na dinâmica de doenças infecto-contagiosas. Esses estudos englobam variações geográficas, temporais, sociais, ecológicas, e visam compreender diversos cenários que representem distintas situações, dentre as quais as epidemiológicas. Justificativas para o emprego de modelos baseados em indivíduos são a inerente natureza estocástica de certos eventos; a dificuldade no tratamento analítico para modelagens baseadas em equações de sistemas complexos; a complexidade da modelagem da interação entre indivíduos, e entre indivíduos e o meio ambiente; a heterogeneidade na distribuição espaço-temporal dos indivíduos e no meio; a heterogeneidade do sistema relacional de contatos e a dinâmica evolucionária de tais sistemas e indivíduos [27, 37].

Dentre as soluções a essas questões estão as que provêm de abordagens baseadas em indivíduos e em redes de contato, que tratam cada indivíduo da comunidade ou da região individualmente. Mesmo essas abordagens, cujos exemplos são os autômatos celulares e os agentes computacionais, embutem em suas especificações questões básicas que fundamentam 
a elaboração dos tradicionais modelos baseados em equações. Entre elas estão as estruturas compartimentais que são obtidas pela subdivisão da população em categorias, entre as quais os indivíduos fluem de acordo com as características da doença e das relações entre os diversos cenários.

Neste trabalho são apresentados e discutidos alguns fundamentos que embasam o estudo da dinâmica espaço-temporal de modelos computacionais epidemiológicos. Em sequência é enfocado o modelo SIR, sendo apresentadas e discutidas modelagens utilizando-se distintas abordagens à tal modelo compartimental. Quando convenientemente parametrizadas elas fornecem soluções qualitativamente assemelhadas.

\subsection{Caracterizações Relevantes à Modelagem}

Epidemiologia pode ser definida como o estudo da frequência, da distribuição e dos determinantes dos estados ou eventos relacionados com a distribuição de doenças transmissíveis e não transmissíveis em populações específicas, e a aplicação dos resultados desses estudos na prevenção e controle dos problemas de saúde [26].

Doenças transmissíveis têm fases definidas à latência, incubação, infecciosidade e recuperação da infecção, entre outras, que são determinantes na construção de modelo a representação de cenário endêmico ou epidêmico. Pode-se perguntar se é possível construir modelos computacionais capazes de reproduzir padrões dinâmicos complexos como a persistência de doenças nos períodos inter-epidêmicos, que são a sucessão de epidemias intercaladas por períodos de pequeno número de casos, e as oscilações não-aleatórias e sustentadas na incidência e no impacto da vacinação. Essas questões são tratadas em [4, 38, 21, 5, 3], que discutem conceitos relevantes, entre eles os de sustentabilidade da infecção; número reprodutivo básico; força de infecção; imunização de fração da população; matrizes de contato; análise de sensibilidade e incertezas; distribuição de idades e de fertilidade; variações demográficas, transmissão dependente da densidade, transmissão dependente da frequência; limiar de transmissão; mobilidade humana; dinâmica espacial; tipos de mistura e a estruturação da população.

Deve-se decidir quais dessas características são de interesse, e então determinar se o modelo será do tipo determinístico ou estocástico, discreto ou contínuo, com população homogênea ou heterogênea, e assim por diante. A escolha pode não ser simples uma vez que a modelagem, ao abstrair um problema complexo a um outro mais tratável, inevitavelmente simplifica características do problema original. Geralmente é difícil assegurar se o modelo resultante é ou não suficientemente acurado, antes que dados sejam obtidos e utilizados para calibração e validação do modelo. 


\subsection{Modelos Compartimentais}

Uma modelagem relacional entre elementos de sistema busca representar as interações decorrentes das suas caracterizações. A abordagem usualmente empregada é a de diagrama de blocos ou em compartimentos. Doenças infecciosas admitem uma modelagem em estrutura compartimental, obtida por meio da divisão da população em categorias, entre as quais os indivíduos fluem com taxas que dependem das características da dinâmica espaço-temporal da doença [9]. Supondo que $N(t)$ designa a quantidade de indivíduos num nível de tempo $t$ e tomando uma modelagem compartimental, os indivíduos que compõem a população podem ser divididos em classes do tipo suscetíveis, $S(t)$; latentes ou expostos, $E(t)$; infectados, $I(t)$, e removidos, $R(t)$, entre outras.

Cada indivíduo pode estar em somente um desses estados em um dado período de tempo. O estado suscetível indica que o indivíduo ainda não contraiu a doença e está apto a adquiri-la. O estado exposto indica que o indivíduo contraiu a doença, mas não pode transmiti-la. O estado infectante indica que o indivíduo está infectado com a doença, sendo capaz de transmiti-la para outros que estejam no estado suscetível, e o estado removido indica que o indivíduo já foi infectado com a doença e não pode se contaminar novamente nem transmiti-la. Considerando-se os estados relacionados com o processo infeccioso, modelos usuais são o Suscetível-Infectado-Suscetível, SIS, empregado em situações em que a doença não confere imunidade, passando o indivíduo de infectado para suscetível novamente, e o modelo Suscetível-Infectado-Removido, SIR, para casos de doenças em que os indivíduos podem se recuperar e adquirir imunidade permanente.

Um artigo basilar à modelagem epidemiológica é o de [19], por meio do qual os autores estabeleceram as bases conceituais e matemáticas de um modelo compartimental SIR. Tal modelo é amplamente estudado e serve como referencial para o estudo e a validação de novas abordagens, visto que modelos SIR possuem uma dinâmica suficientemente atrativa aos estudos, e uma análise matemática sofisticada quando modelado em equações diferenciais, além de possuir vasta literatura técnica a respeito [28, 12]. Não obstante as inerentes limitações de modelos matemáticos baseados em equações à simulação de processos epidemiológicos, eles fornecem uma base aos modelos em indivíduos, que também utilizam nas suas modelagens conceitos e processos análogos [8]. Além das questões subjacentes à modelagem compartimental, as análises matemáticas realizadas com modelos determinísticos podem ser empregadas para estabelecer algumas especificações à modelos baseados em indivíduos.

Modelos do tipo SIR são estabelecidos pela divisão da população em grupos de indivíduos suscetíveis, $S(t)$, infectados, $I(t)$ e removidos, $R(t)$. São empregados para descrever surtos epidêmicos supondo que a população é homogênea, suficientemente grande, e que não ocorrem nascimentos, mortes e migrações no período. A transmissão da doença é modelada supondo que a força de infecção, $\lambda$, é proporcional à fração de infectados, isto é, 
$\lambda=\rho I / N$, sendo $\rho$ o número médio de contatos de um indivíduo infeccioso, por unidade de tempo. Toma-se como constante a taxa de recuperação, $\delta$, dos indivíduos.

Se o modelo SIR é empregado em situações endêmicas, de período mais longo e durante o qual existe a renovação de indivíduos suscetíveis pelo nascimento ou pelo término de eventuais imunizações temporárias, é conveniente que se modele a ocorrência de nascimentos e mortes, cujas taxas, $\gamma$, são tomadas algumas vezes como iguais para simplificar a modelagem. A Figura 1 (a), ilustra uma representação para os compartimentos e as direções dos fluxos de indivíduos do modelo SIR endêmico, enquanto a Figura 11b) ilustra um modelo matemático para tal modelo conceitual, ambos sem imunização temporária, utilizando-se de usuais notações e representações matemáticas [12].

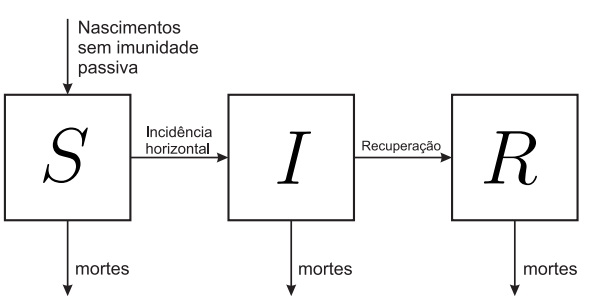

(a) Formulação compartimental.

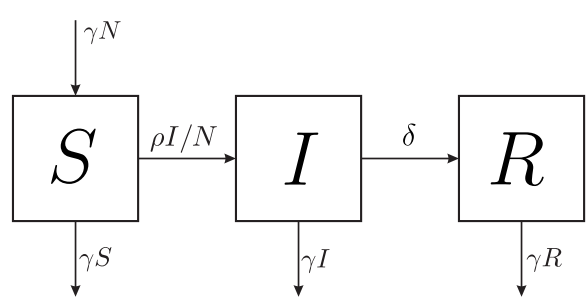

(b) Formulação matemática.

Figura 1: Modelo tipo SIR.

Algumas vezes, por conveniência na análise e nas discussões, pode-se representar as quantidades envolvidas em termos de proporções. Para uma população que é constante de tamanho $N \neq 0$ escreve-se $S(t)+I(t)+R(t)=N$ e então $S(t) / N+I(t) / N+R(t) / N=1$ obtendo $s+i+r=1$, sendo que $s=S(t) / N, i=I(t) / N$ e $r=R(t) / N$ denotam as frações de indivíduos suscetíveis, infectados e removidos, respectivamente.

\section{Modelagem por Equações Diferenciais}

Supondo que as variáveis envolvidas no processo sejam diferenciáveis em relação ao tempo, $t$, pode-se escrever o modelo matemático SIR como um problema de valor inicial em equações diferenciais ordinárias não-lineares, EDOs, à variável temporal, $t$, em que $t_{0}=0$, 
como a Equação (1).

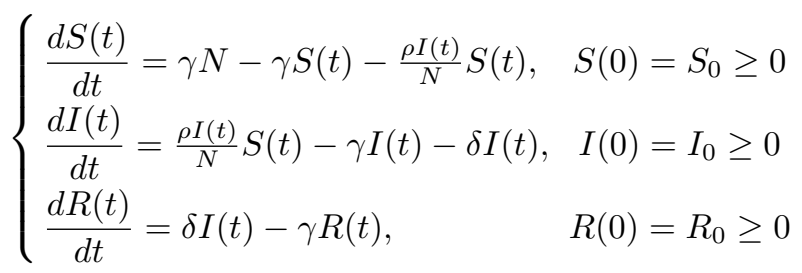

As interpretações epidemiológicas das expressões matemáticas da Equação (1), são de que o termo $\lambda S$ é a incidência horizontal da doença, isto é, a quantidade de novos casos da doença por unidade de tempo, devido aos indivíduos suscetíveis. O parâmetro $\rho I / N=\rho i$ é uma modelagem simplificada à força de infecção exercida pela doença sobre os suscetíveis, tentando infectá-los, sendo a probabilidade, por unidade de tempo, de que um indivíduo suscetível seja contaminado. As taxas de nascimento e de mortalidade foram tomadas como iguais a $\gamma$. A taxa de recuperação $\delta$ representa o fator de indivíduos que deixam a classe dos infectados, por cura ou morte. Neste trabalho não se considera que os indivíduos sejam retirados da simulação por morte, mas apenas que se recuperam com imunização permanente.

Geralmente na modelagem compartimental e determinística se assume que a população é homogênea e o ambiente é uniforme. Porém, atualmente são realizados esforços no sentido de representar os contatos individuais, os efeitos do comportamento individual, a interação entre diferentes tipos de indivíduos e de comunidades, e os aspectos espaço-temporais de tais relações [1, 22, 23, 18, 24, 13, 7]. Algumas abordagens nesse sentido são discutidas a seguir.

\section{Modelagem por Autômato Celular Tipo Difusivo}

Autômatos Celulares, AC, originaram-se nos trabalhos desenvolvidos por John Von Neumann e Stanislaw Ulam nos anos de 1940. Outras importantes e originais contribuições foram dadas por Conway e Wolfram nas décadas de 1970 e 1980, [40], [14]. Autômatos celulares estão presentes numa variedade de simuladores de sistemas físicos e biológicos, entre outros, visto sua capacidade de simular complexas dinâmicas espaço-temporal.

AC podem ser classificados como modelos de sistemas dinâmicos discretos no espaço e no tempo, que operam sobre um lattice contendo uma quantidade finita ou infinita de células. São caracterizados por iterações locais em que cada célula está associada a um estado ou fase por meio de conjunto discreto de valores. A atualização do estado é realizada a partir dos estados das células vizinhas de acordo com regras locais. Autômatos celulares possuem um domínio especificado pelo ambiente ou espaço celular em que estes são operados; um espaço de estados das células em que cada célula do lattice possui determinado estado; a 
vizinhança de cada célula; e a regra de transição local que atua sobre a célula de modo que seu estado pode variar ao ocorrer uma transição.

O modelo aqui discutido inspira-se em [36], em que os autores introduziram um modelo SIR para simular o espalhamento de epidemias e estratégia de vacinação. No artigo de [33] é apresentado um modelo espaço-temporal para descrever a dinâmica da população de vetores da doença de Chagas, dada a necessidade de estabelecer estratégias de controle, pois não existe vacina à doença. Esses autores também investigaram o espalhamento periódico e espacial de doença em localidade da península de Yucatan, no México. Em [15] é apresentada uma abordagem baseada em AC para analisar e estudar padrões espaço-temporais da infecção hantavirus. Em [2] é estudada a influência da heterogeneidade espacial no espalhamento do influenza A. Em [25] e [10] são empregados AC para estudar a persistência e os padrões de espalhamento da dengue, respectivamente.

\subsection{Autômato celular}

O autômato celular é definido sobre um lattice finito com adequada vizinhança às células, sendo sua discretização especificada pelo conjunto $\mathcal{L}=$ $\left\{(i, j) ; 1 \leq i \leq n, 1 \leq j \leq m ; n, m \in \mathbb{N}^{*}\right\}$. A vizinhança da célula $(i, j)$ é do tipo Moore, $\mathcal{V}_{i, j}=\{(i+\alpha, j+\beta) ;-1 \leq \alpha \leq 1,-1 \leq \beta \leq 1 ; \alpha, \beta \in \mathbb{Z}\}$, sendo ela localmente $\mathcal{V}=\{(0,0),(-1,0),(-1,1),(0,1),(1,1),(1,0),(1,-1),(0,-1),(-1,-1)\}$. A vizinhança sem a célula central é indicada por $\mathcal{V}^{*}=\mathcal{V}-\{(0,0)\}$.

A regra de transição local, $\sigma$, é especificada aos estados $\{S, I, R\}=\mathcal{Q}$, de modo que, dados distintos, discretos e consecutivos níveis de tempo $t$ e $t+1$ têm-se $\sigma\left(e_{(i+\alpha, j+\beta)}^{t}\right)=$ $E_{i, j}^{t+1}$, sendo $e_{(i+\alpha, j+\beta)}^{t}$ a designação para o estado de uma célula vizinha da célula $(i, j)$ no nível de tempo $t$, e $E_{i, j}^{t+1}$ a notação para o estado da célula $(i, j)$ no tempo $t+1$, em que $e$ e $E$ são estados quaisquer de $\mathcal{Q}$. Para um espaço celular finito deve-se especificar condições de contorno apropriadas, e algumas delas empregadas em autômato celular são a periódica, tipo reflexiva e a tipo Dirichlet, e um caso particular dessa última, a condição homogênea nula. À especificação da condição de contorno periódica faz-se $E_{1, j}=E_{n, j}$ e $E_{i, 1}=E_{i, m}$ e à condição de contorno homogênea faz-se $E_{1, j}=E_{n, j}=0$ e $E_{i, 1}=E_{i, m}=0$, para tais células em $\partial \mathcal{L}$, o conjunto das células de contorno do domínio.

Assim, AC são formalmente caracterizados pela especificação da quádrupla $\langle\mathcal{L}, \mathcal{Q}, \mathcal{V}, \sigma\rangle$ que atenda as características de homogeneidade espaço-temporal na sua dinâmica, e da especificação de regras de transição não ambíguas. Como as simulações de eventos epidemiológicos requerem uma distribuição dos estados no tempo inicial, $t_{0}$, é necessário especificar tal condição. Escrevendo o AC como um problema de valor inicial e de contorno discreto, a regra de transição é definida em $(i, j) \times t \in \mathcal{L} \times(0, T)$, sendo as condições iniciais dadas $(i, j) \in \mathcal{L}$ para $t_{0}=0$ e as condições de contorno especificadas em 
$(i, j) \times t \in \partial \mathcal{L} \times[0, T]$, sendo que $T$ denota um determinado tempo. Considerando-se as expressões para o domínio e a regra de transição, e tomando a condição de contorno periódica, o AC é como na Equação (2).

$$
\begin{cases}\sigma\left(e_{(i+\alpha, j+\beta)}^{t}\right)=E_{i, j}^{t+1} ; & (i, j) \in \mathcal{V}_{i, j} \subset \mathcal{L}, t>0 \\ E_{1, j}=E_{n, j}, E_{i, 1}=E_{i, m} ; & (i, j) \times t \in \partial \mathcal{L} \times[0, T] \\ e_{(i, j)}^{0}=e(0) ; & (i, j) \in \mathcal{L}\end{cases}
$$

A solução da Equação (2) é obtida por meio da evolução espaço-temporal dada pela regra de transição que fornece os estados finais a partir do cálculo dos estados iniciais. A transição se dá num nível de tempo $t$ para o consecutivo nível de tempo $t+\Delta t$, sendo $\Delta t$ o passo de tempo.

\section{Modelagem por Autômato Celular Tipo LGCA}

Modelos para simular a transmissão de epidemias quase sempre enfatizam a abordagem baseada em equações ordinárias. Essa escolha firmou-se desde os trabalhos originais de [19] e motivos para os quais eles ainda vêm sendo desenvolvidos e aprimorados são como os já discutidos. Soluções à distribuição heterogênea e baseadas em indivíduos modeladas em AC probabilísticos com concepção de movimento molecular são discutidas em [11] e [34].

Esses AC são do tipo Lattice Gas Cellular Automata, LGCA, e Lattice Boltzmann Method, LBM. Em [11] é investigada a dinâmica espaço-temporal de modelo SIR sob modelagem LGCA. Além de pesquisarem o efeito da distribuição espacial inicial não homogênea de infectados e discutirem estratégias de vacinação, os autores especificaram uma distribuição de campo médio para comparar o AC com tais resultados. Em [31], é apresentado um estudo comparativo entre diferentes métodos e abordagens, inclusive à dinâmica SIR, e dentre elas estão o LGCA e Stochastic Cellular Automata, SCA. Embora tais abordagens envolvam diferentes técnicas e tipos de interações espaciais, elas mostram resultados que são qualitativamente consistentes. Outros modelos de difusão e de reação em AC são discutidos em [35].

Na construção do LGCA deve-se especificar uma quádrupla $\langle\mathcal{L}, \mathcal{Q}, \mathcal{V}, \sigma\rangle$ que atenda as características de AC. Supõe-se que a população é compartimentalizada em indivíduos cujo estado $\tau$ é do tipo suscetível, infectado ou removido, sendo $\tau \in\{S, I, R\}=\mathcal{Q}$. São desprezados os estados intermediários, como o período de incubação e de latência. O espaço celular em que os autômatos celulares são operados, o lattice $\mathcal{L}$, tem topologia hexagonal no artigo de [11], mas neste trabalho a escolha recaiu, por conveniência de implementação e 
para efeitos de comparação empírica com outros, em desenvolver um AC numa topologia de células quadradas.

Escrevendo o LGCA como um problema de valor inicial e de contorno, à condição inicial em $t_{0}=0$, os indivíduos que representam os estados $S, I$ e $R$ são distribuídos aleatória e independentemente entre as células de $\mathcal{L}$. Pela metodologia escolhida à movimentação dos indivíduos tal distribuição deve assegurar que existem no máximo quatro indivíduos por célula, independentemente do tipo, visto que se mimetiza indivíduos como partículas sendo movimentadas no ambiente pelas quatro arestas existentes entre as células do lattice, não podendo os indivíduos ocuparem mesma posição na aresta, em um mesmo passo de tempo. No modelo implementado, as condições de contorno são periódicas, indicando que os indivíduos do tipo $\tau$ que chegam até uma célula de contorno de posição, por exemplo, $(n, j)$ emergem na posição $(1, j)$ satisfazendo a condição $\tau_{1, j}^{t}=\tau_{n, j}^{t}$. Não são consideradas as situações de mortes, nascimento e migração de indivíduos da população, e a mistura no LGCA decorre da randomização da direção do movimento dos indivíduos na etapa de randomização $D$ e no movimento dos indivíduos na etapa de propagação $P$. O modelo LGCA é como apresentado na Equação (3).

No modelo da Equação (3), $\tau$ indica um estado qualquer, ou seja, $\tau \in\{S, I, R\}$, e o operador $\tilde{\sigma}$ é um algoritmo do operador $\sigma$. Ou seja, $\tilde{\sigma}=\tilde{P} \circ \tilde{D} \circ \tilde{C}$, sendo $\tilde{P}$ e $\tilde{D}$ procedimentos ou algoritmos para implementar as fases de propagação e de randomização.

$$
\begin{cases}\tilde{\sigma}\left(\tau_{(i+\alpha, j+\beta)}^{t}\right)=\tau_{i, j}^{t+1} ; & (i, j) \in \mathcal{V}_{i, j} \subset \mathcal{L}, t>0 \\ \tau_{1, j}=\tau_{n, j}, \tau_{i, 1}=\tau_{i, m} ; & (i, j) \times t \in \partial \mathcal{L} \times[0, T] \\ \tau_{(i, j)}^{0}=\tau(0) ; \quad(i, j) \in \mathcal{L}\end{cases}
$$

A evolução espaço-temporal do LGCA da Equação (3) é realizada a cada passo de tempo e um operador de evolução é aplicado simultânea e independentemente em todas as células do lattice. Esse operador de evolução é a regra de transição local, $\sigma$, que atua sobre as células cujos estados podem ou não variar para outro num nível de tempo discreto. É especificado por uma composição das operações de contato, $C$, randomização, $D$, e propagação, $P$, como $\sigma=P \circ D \circ C$ [11]. Na operação de contato supõe-se que os indivíduos residem no interior da célula do lattice e se movem somente por meio das suas arestas, e que cada indivíduo suscetível numa célula, independentemente de outros, têm igual probabilidade de serem infectados. Se a $p_{i, j}$ indica a probabilidade de ocorrência do evento na célula $(i, j)$, cada suscetível pode se tornar infectado com probabilidade $\left(1-\left(1-p_{i, j}\right)^{N_{I}}\right)$, em que $N_{I}$ é a quantidade de indivíduos infectados numa célula [11].

A operação de randomização é aplicada a cada célula independentemente de outra, e a população de indivíduos na célula é randomicamente distribuída. O processo de 
redistribuição de indivíduos na célula é probabilístico. Na operação de propagação, os indivíduos se movem simultaneamente de suas células $(i, j)$ às vizinhas pelas arestas. É por meio das arestas, permitindo-se apenas um indivíduo por aresta por passo de tempo, que os indivíduos se movem nessa fase de propagação da célula, desde que a posição correspondente da célula vizinha esteja desocupada, visto que mimetiza-se a ideia de colisão de partículas.

\section{Modelagem por Multiagente}

Agentes baseados em modelos apresentados neste trabalho são definidos espaço-temporalmente especificando-se como ocorre a transição do seu estado num intervalo de tempo e seu movimento no ambiente, de uma posição para outra no passo de tempo, em um ciclo de transição. A especificação formal do agente é realizada por meio de um operador de evolução que define o estado atual do agente, quando interagindo com o ambiente, que decorre da composição do operador de transição temporal, que realiza a transição do estado do agente de acordo com sua interação com outros agentes e com o ambiente, e o operador de transição espacial, que movimenta o agente de sua posição, considerando sua conectividade e mobilidade.

Para a dinâmica são consideradas as operações de movimentação, contato e de transição de estados. Na movimentação, os indivíduos transitam no ambiente matricial por meio de suas vizinhanças de Moore às posições escolhidas aleatoriamente, respeitando os limites espaciais do ambiente. Nas operações de contato ocorre, probabilisticamente, a transmissão da doença por meio dos indivíduos infectados para os indivíduos suscetíveis que ocupam mesma posição no ambiente. Nas transições de estados ocorrem a passagem de indivíduos suscetíveis para expostos, e de expostos para infectantes, e de infectantes para removidos, nessa sequência e uma vez a cada ciclo.

Um agente $\chi(t)$ é definido como $\chi(t)=(P, C, E)$, em que $P$ é a sua posição no ambiente, $E$ designa o seu estado, e $C$ um registro para o contador de ciclos que controla as transições de estados [30]. Cada agente implementa a operação de evolução que atualiza seu estado atual quando interagindo com o ambiente, sendo ele definido pela composição entre os operadores $\mu$ e $\sigma$. O operador de conecto-mobilidade, $\mu$, movimenta o agente de sua posição considerando-se os atributos de conectividade e mobilidade, e o operador $\sigma$ realiza a transição do estado do agente por meio de sua interação com outros agentes e o ambiente.

O operador espaço-temporal $\lambda(\chi(t))$ realiza as operações do agente $\chi(t)$ movimentando-o da posição $(i, j)$ para uma posição $(\xi, \eta)$ no ciclo de tempo, $t$, para o ciclo de tempo, $t+\Delta t$. Formalmente, o estado do agente $\chi(t)$ é representado por um problema de valor inicial e de contorno, como na Equação (4), sendo que $\partial \mathcal{M}$ designa o contorno do domínio e $\mathcal{M}$ o domínio matricial dos indivíduos, o ambiente em que ocorre a dinâmica 
multiagente.

$$
\left\{\begin{array}{l}
\lambda(\chi(t))=\sigma(\mu(\chi(t))) \\
\lambda_{1, j}=\lambda_{n, j}, \lambda_{i, 1}=\lambda_{i, m} ;(i, j) \times t \in \partial \mathcal{M} \times[0, T] \\
\lambda_{(i, j)}^{0}=\lambda(0) ; \quad(i, j) \in \mathcal{M}
\end{array}\right.
$$

Em [20] podem ser encontradas discussões sobre modelagens e análise matemática de sistemas multiagentes, enfocando modelos que sejam simples, mas com baixo custo computacional e pouca demanda por comunicação, sendo, além disso, robustos, podendo se recuperar rapidamente de falhas, e adaptáveis às mudanças no ambiente. Tais tipos de sistemas podem ser analisados matematicamente, podendo-se determinar quais estratégias o agente deve considerar para apresentar o comportamento coletivo desejado.

\section{Experimentos Computacionais e Resultados}

Nesta seção são discutidos os modelos tipo SIR para as Equações (1), (2), (3) e (47. Referências adicionais a tais abordagens são [17, 16, 36, 11]. O enfoque do estudo é numérico-computacional e busca mostrar as diferenças significativas entre as soluções de modelos tipo SIR às distintas abordagens. Visando simplificar o estudo tomou-se o modelo com taxas de natalidade e de mortalidade nulas, ou seja, $\gamma=0$, na Equação (1).

\subsection{Especificação e Parametrização em EDO da Equação (1).}

O problema de valor inicial da Equação (1) é escrito na forma semi-discreta como na Equação (5), cuja solução tem comportamento característico da abordagem utilizada, sendo o tempo, $t$, a única variável independente envolvida no processo.

$$
\left\{\begin{array}{l}
\frac{d S}{d t}=\gamma N-\gamma S^{t}-\frac{\rho I^{t}}{N} S^{t}, \\
\frac{d I}{d t}=\frac{\rho I^{t}}{N} S^{t}-\gamma I^{t},-\delta I^{t} \\
\frac{d R}{d t}=\delta I^{t}-\gamma R^{t},
\end{array}\right.
$$

O modelo da Equação (5) é resolvido por clássicos métodos de Runge-Kutta para equações diferenciais ordinárias, que estão implementados em diferentes pacotes de solução, a exemplo do software Scilab [32]. A função $O D E$ do Scilab emprega abordagens para matrizes rígidas e não rígidas, com o método preditor-corretor de Adams, e expressões de discretização tipo diferenciação regressiva no tempo. Inicia a solução com métodos para 
sistemas rígidos e monitora dinamicamente a solução para decidir qual método de solução deve ser usado. A Figura 2 ilustra a solução do modelo em EDO para os dados utilizados.

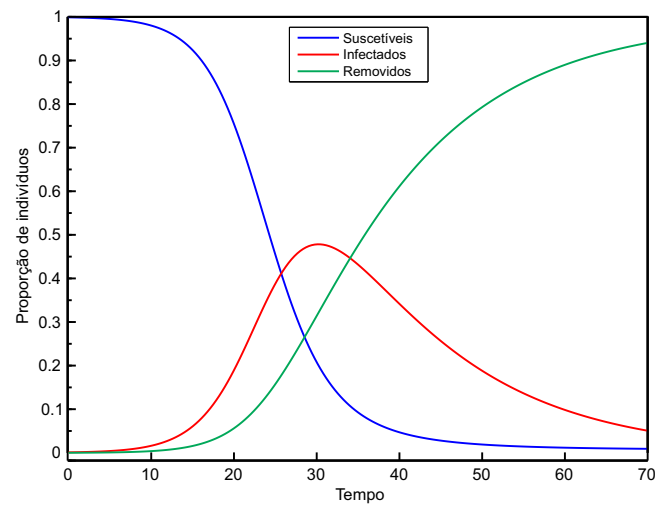

Figura 2: Curvas populacionais do modelo SIR.

O resultado foi obtido empregando como parâmetros as frações $S_{0}=0.999, I_{0}=$ 0.001 e $R_{0}=0.0$ às condições iniciais à solução do sistema dado na Equação (5), com $\gamma=0, \lambda=0.35, \delta=0.07$ e tempo de simulação de 70 passos.

\subsection{Especificação e Parametrização da Equação (2):}

Para simulação utilizam-se proporções de indivíduos suscetíveis, infectados e removidos em todas as células, em que $s_{i, j}^{t} \in[0,1], i_{i, j}^{t} \in[0,1]$ e $r_{i, j}^{t} \in[0,1]$, representam as frações da população suscetível, infectante e removida, na célula $(i, j)$, no instante $t$. A vizinhança $\mathcal{V}$ da célula $(i, j)$ é especificada pelas células $(i+\alpha, j+\beta)$ para todo $(\alpha, \beta) \in \mathcal{V}$. $\mathrm{O}$ fator de conectividade entre tais células e a célula $(i, j) \operatorname{com} \alpha, \beta \in \mathcal{V}^{*}$, é designado por $c_{\alpha, \beta}^{(i, j)} \in[0,1]$. O fator de movimentação entre essas células é designado por $m_{\alpha, \beta}^{(i, j)} \in[0,1]$. Tais parâmetros são interpretados como a proporção de indivíduos que, desejando deslocar-se de uma célula a outra, efetivamente conseguem fazê-lo. Em estudos de caso de [36], $c_{\alpha, \beta}^{(i, j)}$ assume os valores que dependem dos meios de transporte existentes entre as células, e o parâmetro $m_{\alpha, \beta}^{(i, j)} \in[0,1]$ representa a probabilidade de que um indivíduo infectado pertencente à célula $(i+\alpha, j+\beta)$ se desloque à célula central $(i, j)$.

Sendo assim, os fatores de conectividade e movimento dependem da infraestrutura de transporte entre células, e da quantidade de indivíduos infectados, respectivamente, podendo variar de acordo com as características do ambiente e da doença. A taxa de virulência $v \in[0,1]$ indica a proporção de contatos adequados em relação a quantidade de contatos 
entre indivíduos suscetíveis e infectados. A taxa de recuperação $\varepsilon \in[0,1]$ representa a proporção de indivíduos infectados que se recuperam da doença em cada instante de tempo. Para efeito de modelagem, a taxa de virulência foi agrupada com os fatores de conectividade e mobilidade no parâmetro $\mu_{\alpha, \beta}^{(i, j)}=c_{\alpha, \beta}^{(i, j)} m_{\alpha, \beta}^{(i, j)} v$. Para população constante tem-se $s_{i, j}^{t}+i_{i, j}^{t}+r_{i, j}^{t}=1$, com condições iniciais $i_{i, j}^{0}=i_{0}, s_{i, j}^{0}=s_{0}$ e $r_{i, j}^{0}$. O modelo é como a Equação (6).

$$
\left\{\begin{array}{l}
i_{i, j}^{t}=(1-\varepsilon) i_{i, j}^{t-1}+v s_{i, j}^{t-1} i_{i, j}^{t-1}+s_{i, j}^{t-1} \sum_{(\alpha, \beta) \in \mathcal{V}^{*}} \frac{N_{i+\alpha, j+\beta}}{N_{i, j}} \mu_{\alpha, \beta}^{(i, j)} i_{i+\alpha, j+\beta}^{t-1} \\
s_{i, j}^{t}=s_{i, j}^{t-1}-v s_{i, j}^{t-1} i_{i, j}^{t-1}-s_{i, j}^{t-1} \sum_{(\alpha, \beta) \in \mathcal{V}^{*}} \frac{N_{i+\alpha, j+\beta}}{N_{i, j}} \mu_{\alpha, \beta}^{(i, j)} i_{i+\alpha, j+\beta}^{t-1} \\
r_{i, j}^{t}=r_{i, j}^{t-1}+\varepsilon i_{i, j}^{t-1}
\end{array}\right.
$$

Os resultados são apresentados na Figura 3 que ilustra a dinâmica da simulação. Tomou-se o Lattice do AC, $\mathcal{L}$, com dimensões $50 \times 50$ células, e estado inicial $\left(s_{(25,25)}^{0}, i_{(25,25)}^{0}, r_{(25,25)}^{0}\right)=(0.7,0.3,0)$. A população é homogênea, ou seja, $\forall(i, j) \in \mathcal{L}$ tem-se $N_{i, j}^{t}=100$, sendo a taxa de virulência $v=0.35$ e a taxa de recuperação $\varepsilon=0.07$, com um total de $N=250.000$ indivíduos obtidos da distribuição $50 \times 50 \times 100$, com condição inicial $S_{0}=249.970$ e $I_{0}=30$, sendo tomado na célula central $S_{(25,25)}^{0}=70, I_{(25,25)}^{0}=30$ e $R_{(25,25)}^{0}=0$ indivíduos, já que a distribuição é homogênea com 100 indivíduos por célula. Adotou-se, na simulação, que os fatores de conectividade e mobilidade no AC como $c_{\alpha, \beta}^{(i, j)}=1$ e $m_{\alpha, \beta}^{(i, j)}=0.5, \forall(i, j) \in \mathcal{L}$, inclusive na célula central, de modo que, mesmo quando $(\alpha, \beta)=(0,0)$, tem-se mobilidade interna nas células, com valor 0.5.

Como observado na Figura 3 a propagação da epidemia tem um comportamento concêntrico a partir da condição inicial estabelecida no centro do domínio, e embora ela não mostre diretamente as proporções de indivíduos suscetíveis, infectados e removidos ao longo da simulação, ela ilustra o comportamento espacial da população ao longo do processo epidemiológico. Os pixeis em azul escuro indicam a proporção de suscetíveis, os de cor vermelha indicam os infectados e os de cor verde indicam os removidos.

\subsection{Especificação e Parametrização da Equação (3).}

Das regras de contato, de randomização e de propagação, mais as condições de contorno periódica e a inicial, considera-se $\tilde{P}$ e $\tilde{D}$, algoritmos para implementar as fases de propagação e de randomização, respectivamente, sendo o contato determinado pela formulação $\tilde{C}=\left(1-\left(1-p_{i, j}\right)^{N_{I}}\right)$. Assim sendo, o modelo LGCA é como na Equação (7), $\operatorname{com} \tau \in\{S, I, R\}$, sendo $\tilde{\sigma}=\tilde{P} \circ \tilde{D} \circ C$ uma implementação para o operador $\sigma$.

A condição inicial é implementada por uma distribuição dos indivíduos $\tau \in\{S, I, R\}$ utilizando-se a abordagem de embaralhamento, transformando a sequência ordenada numa 


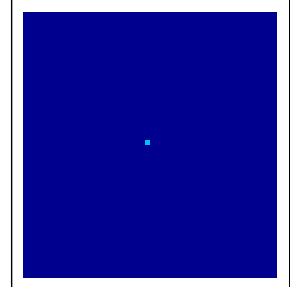

(a) $\mathrm{t}=0$

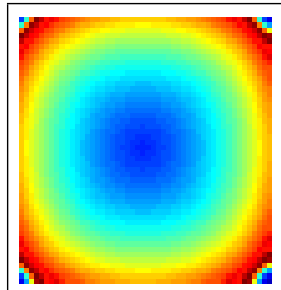

(e) $\mathrm{t}=40$

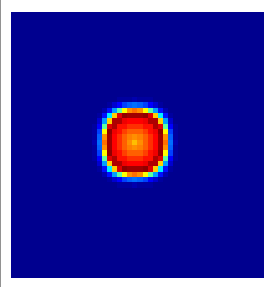

(b) $\mathrm{t}=10$

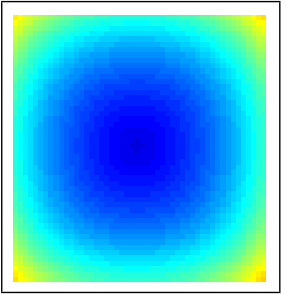

(f) $\mathrm{t}=50$

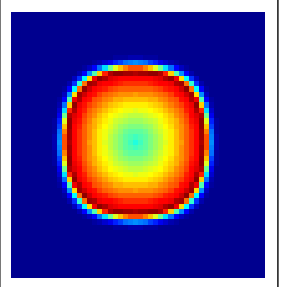

(c) $\mathrm{t}=20$

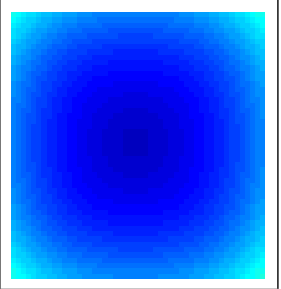

(g) $\mathrm{t}=60$

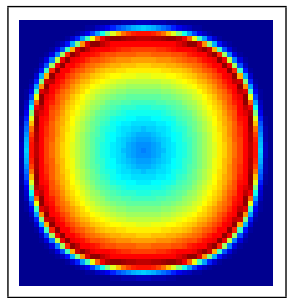

(d) $t=30$

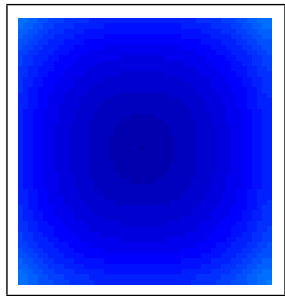

(h) $\mathrm{t}=70$

Figura 3: Dinâmica da Equação (6), com $v=0.35$ e $\varepsilon=0.07,(\alpha, \beta) \in \mathcal{V}^{*}$.

sequência aleatória de indivíduos. O método utilizado é uma versão do algoritmo de Fischer-Yates [39].

$$
\left\{\begin{array}{l}
\tilde{\sigma}\left(\tau_{(i+\alpha, j+\beta)}^{t}\right)=\tau_{i, j}^{t+1} ;(i, j) \in \mathcal{V}_{i, j} \subset \mathcal{L}, t>0 \\
\tau_{1, j}=\tau_{n, j}, \tau_{i, 1}=\tau_{i, m} ;(i, j) \times t \in \partial \mathcal{L} \times[0, T] \\
\tilde{\sigma}\left(\tau_{(i, j)}^{0}\right)=\tau_{(i, j)}^{0} ; \quad(i, j) \in \mathcal{L}
\end{array}\right.
$$

Os parâmetros utilizados em tal modelo incluem o Lattice do AC $\mathcal{L}$ com dimensões $50 \times 50$ células; a vizinhança de Moore $\mathcal{V}$; o estado inicial $\left(s_{(i, j)}^{0}, i_{(i, j)}^{0}, r_{(i, j)}^{0}\right)$, população homogênea no AC randomicamente distribuída; a taxa de infecção $p=0.55$ e a taxa de recuperação $a=0.025$. Resulta que a população é constante com $N=2.070$ indivíduos, com condição inicial de $S_{0}=2.000, I_{0}=70$ e $R_{0}=0$ indivíduos, distribuídos utilizando-se consecutivas vezes o algoritmo de Fischer-Yates até que todos eles estejam distribuídos. 
Autômatos Celulares e Sistema Multiagente para Simulação da Propagação de Doenças

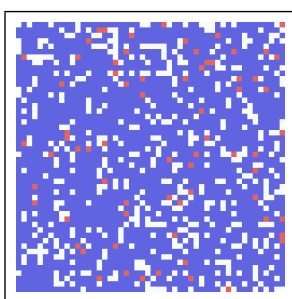

(a) $\mathrm{t}=0$

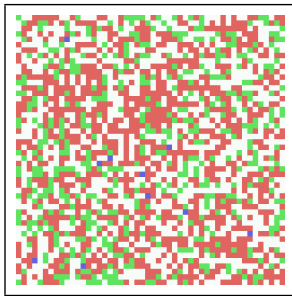

(e) $\mathrm{t}=40$

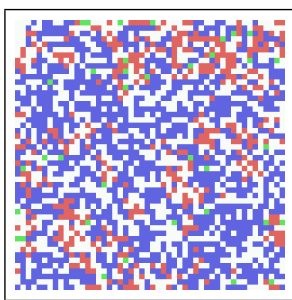

(b) $\mathrm{t}=10$

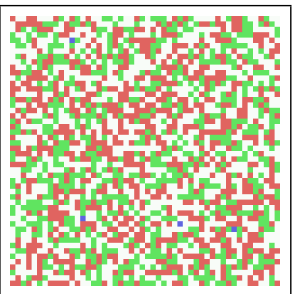

(f) $\mathrm{t}=50$

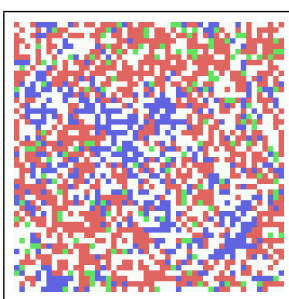

(c) $t=20$

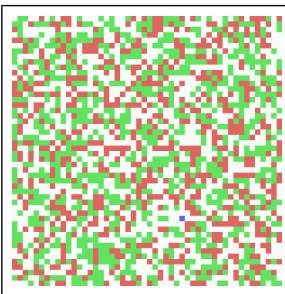

(g) $\mathrm{t}=60$

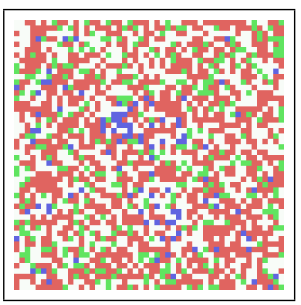

(d) $t=30$

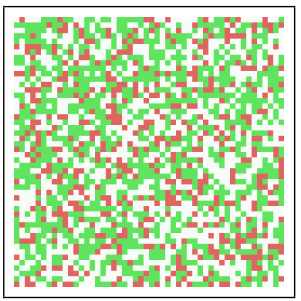

(h) $\mathrm{t}=70$

Figura 4: Dinâmica da Equação (7), $\operatorname{com} p=0.55$ e $a=0.025,(i, j) \in \mathcal{V}^{*}$.

Os resultados ilustrados na Figura 4 mostram que a propagação é dispersiva com efeitos não locais e comportamento emergente observado por meio de agrupamentos de indivíduos não deliberadamente modelado. E, à representação visual dos resultados, é estabelecido que se a célula contém indivíduo infectado, ela é representada na cor vermelha, independentemente de outros indivíduos. Se a célula tem mais indivíduos suscetíveis do que removidos ela é representada na cor azul, independentemente dos espaços não ocupados. Não ocorrendo esses casos, se a célula contém mais indivíduos removidos do que suscetíveis ou se a quantidade de removidos é maior do que zero, é representada na cor verde. Caso essas situações não aconteçam, a célula é representada na cor branca.

\subsection{Especificação e Parametrização da Equação (4).}

O ambiente computacional é composto por uma estrutura matricial com \#L linhas e \#C colunas, tendo uma estrutura de vizinhança tipo Moore para efeitos de conecto-mobilidade do agente que está numa posição $(i, j)$ à uma posição $(\xi, \eta)$ vizinha. O modelo é como a Equação [8].

$$
\lambda\left(\chi(t)_{(i, j)}\right)=\sigma\left(\mu(\chi(t+1))_{(\xi, \eta)}\right)
$$


Os parâmetros pertinentes à simulação, como as quantidades iniciais de agentes e as taxas são definidas em faixas, o que significa que cada parâmetro possui um valor mínimo e máximo. Para as operações que dependem das taxas, ou da movimentação, são randomizados valores dentro da sua faixa ou da posição a ser movimentada para estabelecer o caráter probabilístico do modelo.

Não existem restrições à quantidade de agentes que ocupam mesma posição, quando da distribuição inicial dos agentes ou sua movimentação ao longo da simulação. E os parâmetros às operações são $0.80=\rho_{\min } \leq \rho \leq \rho_{\max }=0.90$ e $20=\delta_{\min } \leq \delta \leq$ $\delta_{\max }=40$, as taxas de infecção e recuperação, respectivamente. Mas a escolha é arbitrária, podendo ser adequada às doenças em que tais parâmetros sejam conhecidos. Foi tomado a parametrização que $\# L=294, \# C=294$, com quantidades iniciais de agentes de $S_{0}=18.800, I_{0}=1.200, R_{0}=0$. A Figura 5 ilustra a dinâmica da simulação desse modelo com condição de contorno tipo reflexiva.

Foi empregado em sua solução a estratégia de Monte Carlo à obtenção dos valores médios das simulações, que consiste na execução de determinada quantidade de simulações com mesma configuração inicial às quantidades de agentes e à dimensão do ambiente [6]. Para cada simulação Monte Carlo é executada, em cada ciclo da simulação individual, as operações de movimentação de agentes no ambiente; a realização dos contatos entre os agentes; e a realização da transição de estados dos agentes. 


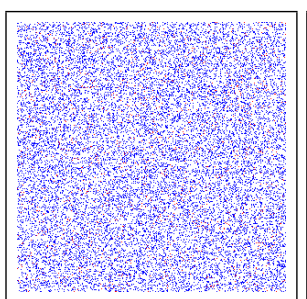

(a) $\mathrm{t}=0$

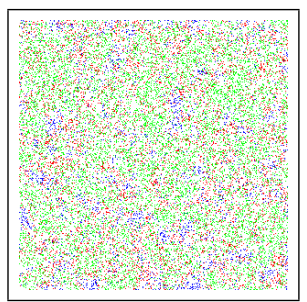

(e) $\mathrm{t}=40$

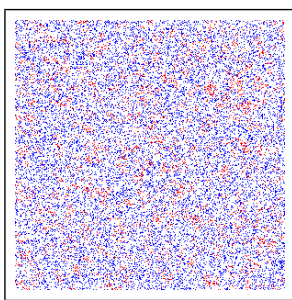

(b) $\mathrm{t}=10$

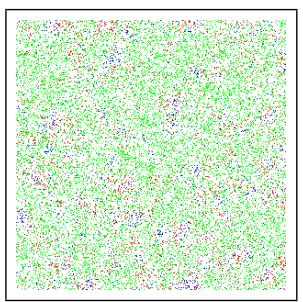

(f) $\mathrm{t}=50$

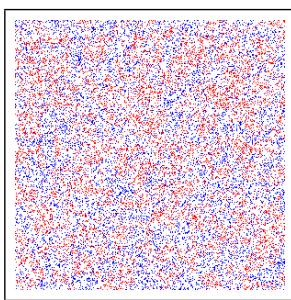

(c) $t=20$

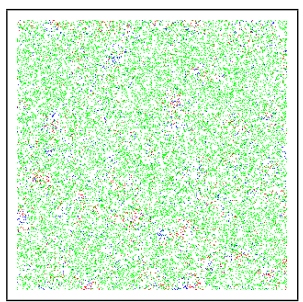

(g) $t=60$

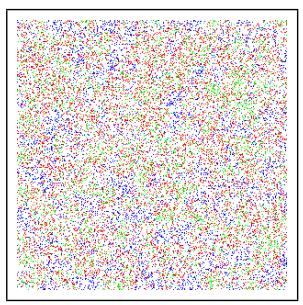

(d) $\mathrm{t}=30$

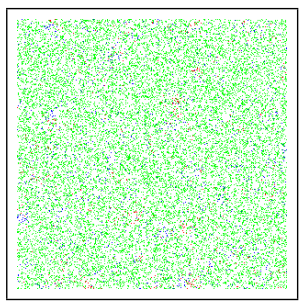

(h) $\mathrm{t}=70$

Figura 5: Dinâmica espaço-temporal do modelo multiagente [30].

\subsection{Comparação entre os modelos}

Não é evidente a comparação direta dos resultados obtidos com as Equações (1), (2), (3) e (4), pois os modelos têm diferentes características. Para viabilizar uma discussão comparativa tomou-se por base a Equação (1) e então foram realizadas parametrizações à dinâmica espaço-temporal visando obter resultados compatíveis com as curvas populacionais do resultado apresentado na Figura 2. Os resultados obtidos em termos de quantidades por estado estão ilustrados na Figura 6 .

Tais resultados foram obtidos em decorrência da conveniente parametrização dos quatro modelos à obtenção das curvas populacionais ilustradas na Figura 6, não significando que os parâmetros utilizados em cada um dos modelos são adequados à específica simulação de mesma doença. Somente após a validação aos dados experimentais que os modelos podem ser empregados para estudar a propagação e a transmissão de doenças. 


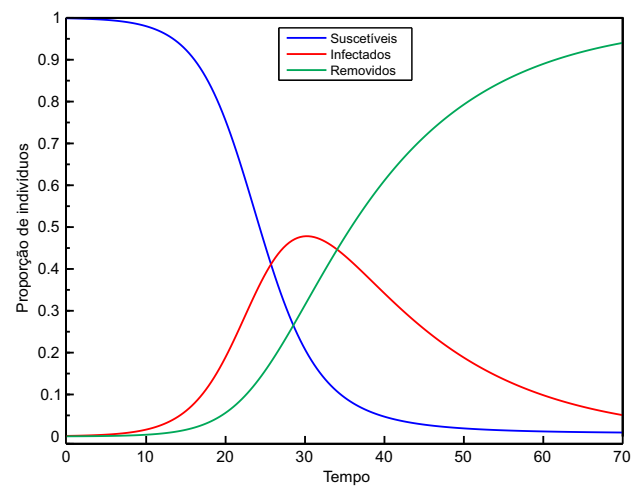

(a) EDO, Equação 5 .

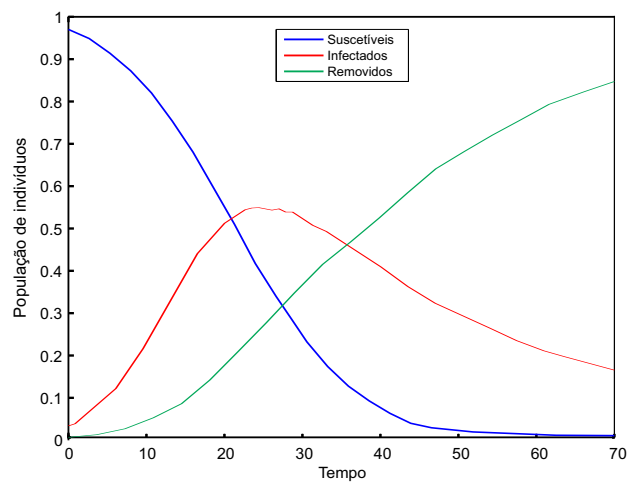

(c) LGCA, Equação (7).

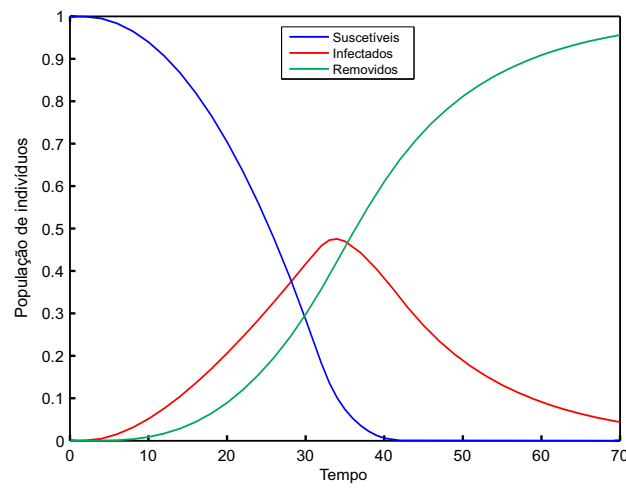

(b) AC difusivo, Equação 6.

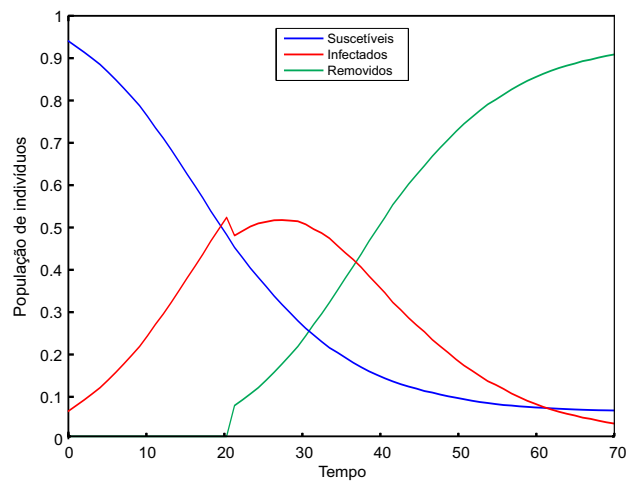

(d) Multiagente, Equação 8 .

Figura 6: Soluções para as abordagens para o modelo compartimental SIR.

Os resultados dos modelos LGCA e multiagentes apresentados na Figura 6 é o valor médio das respectivas soluções, obtidas por meio de 100 execuções, como em uma simulação tipo Monte Carlo. As simulações em EDOs e em AC Difusivo são determinísticas. Como observado na Figura 6, a proporção de indivíduos suscetíveis apresentou um comportamento decrescente para os modelos avaliados. A proporção de indivíduos infectados apresenta um crescimento até atingir uma proporção máxima, entre 25 e 35 passos de tempo, em seguida começa a decrescer, com a redução de indivíduos suscetíveis e incremento da proporção de removidos. Todos os modelos são conservativos para os parâmetros utilizados, em que $s+i+r=1$ em todas simulações. São resultados assemelhados ao de [31], que estudaram modelos em autômatos celulares estocásticos, LGCA e baseado em equações diferenciais 
parciais.

A Tabela 1 sintetiza algumas características de cada metodologia adotada para obtenção da solução dos modelos compartimentais tipo SIR, para propagação e transmissão de determinada doença.

Tabela 1: Características dos modelos apresentados.

\begin{tabular}{|c|c|c|}
\hline Modelo & Possibilidades & Limitações \\
\hline EDO & $\begin{array}{l}\text { Solução eficiente. } \\
\text { Viabiliza uma análise } \\
\text { matemática das soluções. } \\
\text { O pré-processamento à } \\
\text { simulação é mínimo. }\end{array}$ & $\begin{array}{l}\text { Não viabiliza solução espacial. } \\
\text { As soluções são de campo médio. } \\
\text { Não captura o } \\
\text { comportamento individual. } \\
\text { Não viabiliza solução espacial. }\end{array}$ \\
\hline AC-Difusivo & $\begin{array}{l}\text { Viabiliza a solução } \\
\text { espaço-temporal. } \\
\text { Solução eficiente. } \\
\text { É parametrizável às } \\
\text { características do } \\
\text { ambiente. }\end{array}$ & $\begin{array}{l}\text { A solução espacial é difusiva e } \\
\text { não condizente com os dados. } \\
\text { A modelagem é baseada em } \\
\text { visitas às vizinhanças, de forma } \\
\text { que a infecção ocorre devido à } \\
\text { movimentação da onda de infecção e } \\
\text { não dos indivíduos. }\end{array}$ \\
\hline LGCA & $\begin{array}{l}\text { Pode modelar } \\
\text { complexas dinâmicas } \\
\text { espaço-temporais. } \\
\text { É parametrizável às } \\
\text { características do } \\
\text { ambiente. }\end{array}$ & $\begin{array}{l}\text { A eficiência computacional } \\
\text { depende de várias características. } \\
\text { É dependente do formalismo } \\
\text { matemático que se } \\
\text { pretende mimetizar. } \\
\text { A implementação computacional } \\
\text { é mais complexa. }\end{array}$ \\
\hline Multiagente & $\begin{array}{l}\text { É apropriado à modelagem } \\
\text { de complexas dinâmicas } \\
\text { espaço-temporal. } \\
\text { É parametrizável } \\
\text { às características } \\
\text { do ambiente. } \\
\text { É altamente flexível } \\
\text { para inserção de regras à } \\
\text { dinâmica. }\end{array}$ & $\begin{array}{l}\text { A eficiência computacional } \\
\text { dependente de várias características. } \\
\text { A implementação computacional é } \\
\text { mais complexa. }\end{array}$ \\
\hline
\end{tabular}

Na Tabela 1 estão destacadas algumas características de modelos apresentados neste trabalho, que focou o estudo comparativo das referidas abordagens, indicando-se que o tradicional modelo em equações diferenciais ordinárias é relevante para estudos preliminares à dinâmica de doenças infecto-contagiosas, sobretudo na fase de sua modelagem 
e análise matemática de pontos de equilíbrio e de estabilidade da solução, e de outras caracterizações analíticas. O estudo da dinâmica espaço-temporal requer modelos mais versáteis e flexíveis, como os em autômatos celulares e multiagentes. Os tempos de execução para as configurações utilizadas são, aproximadamente, de $t=0.001 \mathrm{~s}, t=0.446 \mathrm{~s}$, $t=0.981 \mathrm{~s}$ e $t=0.811 \mathrm{~s}$, para os modelos em EDO, AC, LGCA e Multiagente, respectivamente.

Observa-se que, embora as soluções sejam qualitativamente assemelhadas e obtidas com parâmetros convenientes, a em Multiagente teve um pico anômalo em torno do ciclo 20 . Não é possível afirmar categoricamente qual situação é a responsável pelo pico. Suposições incluem as taxas de transição, e fato de que os parâmetros epidemiológicos adotados flutuam numa faixa de valores, podendo ter ocorrido que eles se aproximaram dos valores máximos ou mínimos, alterando abruptamente o comportamento da solução. Esse tipo de comportamento é inerente da natureza estocástica do modelo implementado.

\section{Conclusões}

Neste trabalho foi apresentado um estudo comparativo de algumas diferentes abordagens à solução da propagação e transmissão de doenças que possam ser modeladas sob o escopo da abordagem compartimental SIR. Os resultados indicam que a metodologia de Sistema Multiagente tem grande potencialidade para modelar os movimentos, contato e a transição de estados de indivíduos integrantes de Sistemas Dinâmicos Complexos, como aqueles decorrentes de problemas biológicos e epidemiológicos, diferentemente das outras abordagens tratadas neste trabalho.

Para a obtenção de resultados qualitativamente assemelhados com as abordagens estudadas neste trabalho, foi necessária a aplicação de diferentes configurações, seja das quantidades de indivíduos ou nos parâmetros dos modelos, ou em outros elementos característicos de cada modelo. Um trabalho interessante é confrontar os resultados obtidos neste trabalho com aqueles provenientes de tais modelos sob análogas condições, e estudar a sensibilidade dos parâmetros e dos dados, empírica e estatisticamente.

Embora alguns dos modelos apresentados neste trabalho tenham potencial para simulação computacional de processos centrais na dinâmica de doenças infecto-contagiosas, já que eles se mostraram precisos quando convenientemente parametrizados, eles ainda são demasiadamente simples para representar adequadamente um cenário endêmico ou epidêmico. São requeridas modelagens mais sofisticadas à dinâmica espaço-temporal para estudos sérios à sustentabilidade da infecção e estratégias de vacinação, entre outros fatores, para se obter modelos acurados e que se ajustem bem aos dados. Trabalhos nesse sentido estão sendo realizados em [29]. 
Além disso, dada a demanda computacional à simulação de tais eventos em ambientes mais próximos aos dos reais, eles requerem soluções computacionais mais sofisticadas, como aquelas obtidas em ambientes paralelos ou distribuídos. Experimentos computacionais já realizados em [30] indicam que a dinâmica da propagação da doença, assim como a eficiência computacional, são influenciadas significativamente pela quantidade de indivíduos, pela dimensão do ambiente de resolução, pela distribuição da população e pelas estruturas de dados utilizadas, entre outros fatores.

\section{Agradecimentos}

Os autores agradecem aos revisores que por meio de sugestões e comentários, contribuíram para o aprimoramento do trabalho.

\section{Contribuição dos autores:}

Os autores contribuíram igualmente na realização deste trabalho.

\section{Referências}

[1] J. Arino, J. R. Davis, D. Hartley, R. Jordan, J. M. Miller, and P. van den Driessche. A multi-species epidemic model with spatial dynamics. Mathematical Medicine and Biology, 22(2):129-142, 2005.

[2] C. Beauchemin, J. Samuel, and J. Tuszynski. A simple cellular automaton model for influenza a viral infections. Journal of Theoretical Biology, 232(2):223-234, 2005.

[3] M. Begon. Ecological epidemiology. In The Princeton guide to ecology, pages 220-226, 2009.

[4] S. M. Blower and A. R. Mclean. Mixing ecology and epidemiology. In Proceedings: Biological Sciences, volume 245, pages 187-192, 1991.

[5] D. Brockmann. Human Mobility and Spatial Disease Dynamics, pages 1-24. Wiley-VCH Verlag GmbH \& Co. KGaA, 2010.

[6] L. Chwif and A. C. Medina. Modelagem e Simulacao de Eventos Discretos: Teoria e Aplicacoes. Elsevier Brasil, 2014.

[7] B. Cisse, S. Yacoubi, and S. Gourbiere. The spatial reproduction number in a cellular automaton model for vector-borne diseases applied to the transmission of chagas disease. Simulation: Transactions of the Society for Modeling and Simulation International, 92(2):141-152, 2016. 
[8] P. G. Coen. How mathematical models have helped to improve understanding the epidemiology of infection. Early Human Development, 83(3):141-148, 2007.

[9] L. Edelstein-Keshet. Mathematical Models in Biology. Society for Industrial and Applied Mathematics, Philadelphia, PA, USA, 2005.

[10] P. Eosina, T. Djatna, and H. Khusun. A cellular automata modeling for visualizing and predicting spreading patterns of dengue fever. Tetlkomnika., 14(1):228-237, 2015.

[11] H. Fuks and A.T. Lawniczak. Individual-based lattice model for the spatial spread of epidemic. Discrete Dynamics in Nature and Society, 6:191-200, 2001.

[12] H. W. Hethcote. The mathematics of infectious diseases. SIAM Rev., 42(4):599-653, December 2000.

[13] A. Holko, M. Medrek, Pastuszak Z., and Phusavat K. Epidemiological modeling with a population density map-based cellular automata simulation system. Expert Systems With Applications, (48):1-8, 2016.

[14] A. Ilachinski. Cellular Automata: A Discrete Universe. World Scientific Publishing, 2001 .

[15] A. Karim, M. Faisal, M. Ismail, A. Izani, and H. B. Ching. Cellular automata modelling of hantarvirus infection. Chaos, Solitons and Fractals, 41(5):2847-2853, 2009.

[16] M. J. Keeling, O. Bjornstad, and B. T. Grenfell. Metapopulation dynamics of infectious diseases. In Ecology, Genetics and Evolution, pages 415-446, 2004.

[17] M. J. Keeling and P. Rohani. Estimating spatial coupling in epidemiological systems: a mechanistic approach. Ecology Letters, 5(1):20-29, 2002.

[18] M. J. Keeling and P. Rohani. Modeling Infectious Diseases in Humans and Animals. Princeton University Press, 2008.

[19] W. O. Kermack and A. G. McKendrick. A contribution to the mathematical theory of epidemics. Proceedings of the Royal Society of London A: Mathematical, Physical and Engineering Sciences, 115(772):700-721, 1927.

[20] K. Lerman and M. Mataric. Mathematical modeling of multi-agents systems, 2004.

[21] H. McCallum, N. Barlow, and J. Hone. How should pathogen transmission be modelled? Trends in Ecology \& Evolution, 16(6):295-300, 2001.

[22] R. K. McCormack. Multi-host and multi-patch mathematical epidemic models for disease emergence with applications to hantavirus in wild rodents. $\mathrm{PhD}$ thesis, Doctor of Philosophy in Mathematics, 2006. 
[23] R. K. McCormack and L. J. S. Allen. Stochastic sis and sir multihost epidemic models. In Proceedings of the Conference on Differential and Difference Equations and Applications, pages 775-785, 2006.

[24] R. K. McCormack and L. J. S. Allen. Multi-patch deterministic and stochastic models for wildlife diseases. Journal of Biological Dynamics, 1(1):63-85, 2007.

[25] L. C. de C. Medeiros, C. A. R. Castilho, C. Braga, W. V. Souza, L. Regis, and A. M. V. Monteiro. Modeling the dynamic transmission of dengue fever: Investigating disease persistence. PLoS Neglected Tropical Diseases., 5(1), 2011.

[26] R. A. Medronho. Epidemiologia. Editora Atheneu, Sao Paulo - SP, 2009.

[27] M. A. Nowak. Evolutionary Dynamics: exploring the equations of life. Harvard University Press, 2006.

[28] S. Pathak, A. Maiti, and G. P. Samanta. Rich dynamics of an sir epidemic model. Nonlinear Analysis: Modelling and Control, 15(1):71-81, 2010.

[29] R. L. Rizzi, C. B. Rizzi, and W. L. Kaizer. Um modelo multiagentes para a dinamica de populacoes de aedes aegypti com populacao humana acoplada com a co-existencia de multiplos sorotipos de dengue e a presenca ou nao da bacteria wolbachia. Technical report, 2015.

[30] R. L Rizzi, C. B. Rizzi, and W. L. Kaizer. Solucoes paralelas de um modelo multiagente em bitstring para simular a propagacao de doencas baseada em uma modelagem compartimental tipo seirs. Technical report, Centro de Ciencias Exatas e Tecnologicas. Universidade Estadual do Oeste do Parana. Cascavel, 2016.

[31] G. Schneckenreither, N. Popper, G. Zauner, and F. Breitenecker. Modelling sir-type epidemics by odes, pdes, difference equations and cellular automata - a comparative study. Simulation Modelling Practice and Theory, 16(8):1014-1023, 2008.

[32] Scilab. Scilab enterprises s. a. s, 2016.

[33] R. Slimi, S. El Yacoubi, E. Dumonteil, and S.Gourbiere. A cellular automata model for chagas disease. Applied Mathematical Modelling, 33(2):1072-1085, 2009.

[34] J. Voroney and A. T. Lawniczak. Construction, mathematical description and coding of reactive lattice-gas cellular automaton. Simulation Practice and Theory, 7(7):657-689, 2000 .

[35] J. R. Weimar. Cellular automata for reaction-diffusion systems. Parallel Computing, 23(11):1699-1715, 1997. Cellular automata. 
[36] S. Hoya White, A. Martin del Rey, and G. Rodriguez Sanchez. Modeling epidemics using cellular automata. Applied Mathematics and Computation, 186(1):193-202, 2007.

[37] L. Willem. Agent-Based Models for Infectious Disease Transmission. PhD thesis, submetted for the joint-degree of doctor of medical science at the Univerrsity of Antwerp and doctor of science at Hasselt University, 2015.

[38] J. R. Williams. Models for the study of infection in populations. 2006.

[39] M. C. Wilson. Random and exhaustive generation of permutations and cycles. cdmtcs research report series. cdmtcs-295, 2007.

[40] S. Wolfram. Cellular automata. Los Alamos Science, (9):2-21, 1983. 